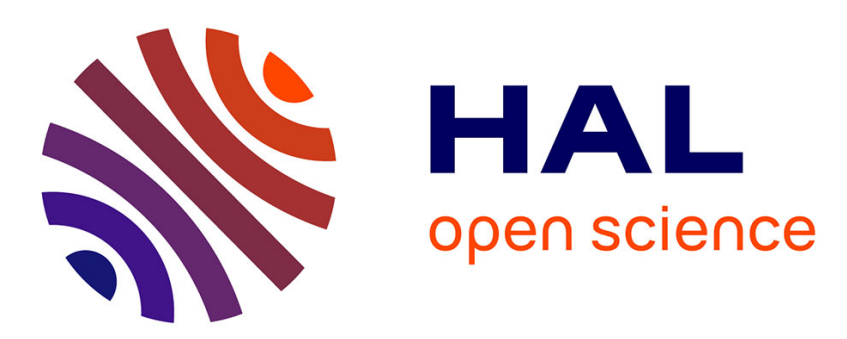

\title{
Les enjeux de la Guerre froide et leurs implications dans un département frontalier : la Meurthe-et-Moselle, 1947-1967
}

Jean-Louis Étienne

\section{- To cite this version:}

Jean-Louis Étienne. Les enjeux de la Guerre froide et leurs implications dans un département frontalier : la Meurthe-et-Moselle, 1947-1967. Sous la direction de Philippe Buton, Olivier Büttner, Michel Hastings. La Guerre froide vue d'en bas, CNRS Editions, p. 191-205, 2014, 9782271078230. halshs01260213

\section{HAL Id: halshs-01260213 \\ https://shs.hal.science/halshs-01260213}

Submitted on 21 Jan 2016

HAL is a multi-disciplinary open access archive for the deposit and dissemination of scientific research documents, whether they are published or not. The documents may come from teaching and research institutions in France or abroad, or from public or private research centers.
L'archive ouverte pluridisciplinaire HAL, est destinée au dépôt et à la diffusion de documents scientifiques de niveau recherche, publiés ou non, émanant des établissements d'enseignement et de recherche français ou étrangers, des laboratoires publics ou privés. 


\section{Les enjeux de la Guerre froide et leurs implications dans un département frontalier : la Meurthe-et-Moselle, 1947-1967}

Jean-Louis Étienne

La forme originale de la Meurthe-et-Moselle résulte des vicissitudes de l'histoire. Ce département, créé en 1871, s'étire de la frontière belgoluxembourgeoise au massif vosgien. L'agriculture y tient une place non négligeable, mais n'occupant pas plus de $7 \%$ des actifs entre 1954 et 1960, alors que $52 \%$ sont mineurs et ouvriers de la sidérurgie et de la métallurgie. L'industrie, anciennement implantée, y est très diversifiée, et ce sont surtout les trois bassins miniers ferrifères et sidérurgiques du Pays Haut (Longwy et Briey) et de Nancy (avec Pompey et Neuves-Maisons) qui créent la richesse : 60 millions de tonnes de minerai de fer sont extraites en 1961. Après la Seconde Guerre mondiale, la reconstruction de l'outil industriel dure une décennie, et la production économique ne retrouve son niveau de 1938 qu'en 1950. Les années 1950-1960 connaissent une forte croissance démographique, résultant d'un solde naturel et d'un solde migratoire positifs : la population augmente ainsi de 528000 habitants en 1946 à 705000 en 1968. Mais le manque de main-d'œuvre nécessite le recours aux immigrés : de 43000 en 1946, les étrangers passent à 56000 en 1962 (surtout Italiens et Polonais). Le chef-lieu, Nancy, est un pôle industriel et tertiaire, qui compte 113000 habitants au lendemain de la guerre (pour la seule ville), et en dénombre 133000 en 1962.

Limitrophe du Reich allemand de 1871 à 1918, et de 1940 à 1945, la Meurthe-et-Moselle a vécu deux périodes d'occupation allemande, et a connu les combats des deux guerres mondiales : c'est un pays de casernes, de fortifications et de cimetières, où les regards sont constamment tournés vers le voisin allemand. Le Lorrain est souvent considéré comme patriote, voire nationaliste, mais d'un nationalisme " défensif », et la Meurthe-etMoselle est une terre républicaine, modérée, laïque, même si l’Église catholique demeure influente auprès des classes moyennes et du monde pay- 
san. Au lendemain de la guerre, la population accorde une large confiance au général de Gaulle, comme en témoigne le succès du " non » au référendum de mai 1946. Les élections suivantes confirment l'avance de la droite et du centre droit. Toutefois, une poussée bien réelle de la gauche cache en fait une « irruption » du communisme. Dans un département qui n’a jamais été une terre socialiste et où le radicalisme disparaît précocement, le parti communiste, qui sort renforcé de sa participation à la Résistance, joue ainsi le rôle de «fondateur de la gauche ». Dès 1945, il obtient un député en la personne du résistant Kriegel-Valrimont, et atteint $24 \%$ des suffrages en novembre 1946. Sans être un département «rouge », la Meurthe-etMoselle présente la particularité de juxtaposer, à une importante majorité modérée et conservatrice de l'opinion, une forte minorité communiste, bien délimitée professionnellement (mineurs, sidérurgistes, cheminots) et géographiquement (Pays Haut). Lorsque surgit la Guerre froide, la population subit encore les séquelles du conflit précédent et reste hantée par le retour d'un danger allemand. La relative proximité avec l'Europe centrale, la présence d'une nombreuse population immigrée, le retour des Américains, plus tard l'arrivée des réfugiés hongrois vont concourir à impliquer l'opinion dans les enjeux de la Guerre froide. Ceux-ci sont-ils clairement perçus? Dans une population d'une grande diversité socio-culturelle, interpellée par la montée du communisme, quelle est donc la réception de la Guerre froide, de ses enjeux politiques et idéologiques?

\section{LE TEMPS DES RUPTURES : COMMENT LE RIDEAU DE FER S'INSTALLE DANS LES ESPRITS}

De quel poids respectif pèsent les événements nationaux et internationaux des années 1947-48 dans la mise en place d'un clivage idéologique amorcé antérieurement avec l'irruption du communisme ? Ce " communisme du fer ${ }^{1}$, dont le nombre de militants est de l'ordre de cinq mille en $1947^{2}$, est de source principalement syndicale, l'adhésion à la CGT précédant en effet le militantisme politique. À la tête de la centrale, le courant ex-unitaire contrôle les syndicats des mines, de la métallurgie et des cheminots. Dès lors, peut-on considérer les grèves de 1947-48, jugées «fondatrices » par certains,

1. Bonnet Serge, L'Homme du fer, mineurs de fer et sidérurgistes lorrains, tome 3, Metz, éd. Serpenoise, 1984. On lira également avec profit, Noiriel Gérard, Longwy. Immigrés et prolétaires, 1880-1981, Paris, PUF, 1984.

2. Le chiffre de 6993, repris des archives du PCF par Roger Martelli, « Prendre sa carte », Fondation G. Péri, 2000, semble excessif. 
comme une tentative de sabotage du plan Marshall ? Il est vrai que seuls le PCF et ses alliés considèrent l'aide américaine comme une «mainmise des trusts anglo-saxons sur l'Europe », dans le but de la « vassaliser ». Les grèves de 1947 sont largement suivies : les sept mines du bassin de Longwy sont touchées. La CGT fait passer les mots d'ordre, mais la discipline syndicale est observée sans enthousiasme, voire avec réticence (refus du vote à main levée). À Longwy, les usines sont paralysées par quatre cents manifestants qui s'empoignent avec les non-grévistes et les forces de l'ordre. Le caractère politique du mouvement n'est pas attesté, même si une enquête policière évoque une circulaire interne postérieure du PCF évoquant «l'éventualité d'une nouvelle grève insurrectionnelle $»^{3}$. Dans son ensemble, la population envisage, au contraire, l'aide américaine avec espoir, dans ces temps de grandes difficultés matérielles, et « la classe populaire ne croit pas à la malignité du plan Marshall $»^{4}$.

En 1948, les événements de Prague et de Berlin contribuent à creuser le fossé idéologique. Si les communistes interprètent le "coup de Prague » comme une « victoire de la démocratie internationale », l'opinion, en accord avec les analyses de la presse régionale, se montre incrédule quant à la version officielle du processus politique et de la mort du ministre Masaryk. Quant aux images filmées du blocus de Berlin, elles ont pour effet de confronter la population à la matérialité d'un rideau de fer, perçu jusqu'alors comme un concept lointain. N'est-il pas distant de seulement 200 kilomètres ? Les grèves de l'automne 1948 touchent les mines et la sidérurgie, et prennent une tournure radicale après l'échec des réunions paritaires. À Villerupt, des accrochages violents opposent six mille manifestants aux forces de l'ordre, tandis qu'à Longwy des cadres sont pris en otages. L'originalité de ces troubles tient à la participation décisive des femmes ; celles-ci, conduites en particulier par l'épouse du leader CGT Marcel Dupont, « une nouvelle passionaria », se montrent « furieuses et d'une folie hystérique ${ }^{5}$. Cette dynamique syndicale explique la montée en puissance du courant communiste, mais n'est pas sans susciter des répliques. Au sein de la CGT, les grèves ne font que concrétiser des tensions préexistantes, qui aboutissent à la création d'un noyau Force ouvrière, autour de René Peeters, ancien président du Comité départemental de Libération. Un congrès départemental fondateur a lieu à Nancy en avril 1948. Les militants non communistes cherchent à évincer les éléments communistes lors des élections professionnelles, ceux-ci reprochant à la tendance FO de saboter les grèves.

3. Archives départementales de Meurthe-et-Moselle (ADMM), RG Longwy, 7 février 1948, W 950/238.

4. La Croix de l'Est, 7 mars 1948.

5. ADMM, Police, 13 novembre 1948, W 1277/205. 
Pour les uns, FO symbolise le "parti américain», pour les autres, la " CGTK » est soumise au Kominform. Au discours communiste s'oppose bientôt le discours du RPF, principal vecteur de l'anticommunisme. Le respect qu'inspire aux Lorrains le général de Gaulle, fait citoyen d'honneur de la ville de Nancy (malgré le désaccord des élus communistes), associé au recul du MRP et au ralliement d'anciens sympathisants de Vichy, conduisent le RPF à des succès électoraux inattendus (23\% des voix aux législatives de 1951). Communistes et gaullistes s'affrontent régulièrement dans le Pays Haut, où selon la police des groupes d'action RPF, équipés d'armes récupérées des FFI, seraient mis sur pied dans deux communes, afin de faire face à une éventuelle « tentative insurrectionnelle » ${ }^{6}$.

Dans ce climat passionnel, "l'étranger », surtout s'il est suspecté d'être communiste, est souvent considéré comme un «ennemi de l'intérieur ». Le cas des Polonais mérite de retenir l'attention. En 1945, sont dénombrés 17000 ressortissants, près de 13000 sont encore présents en 1949, dont 8500 domiciliés dans le seul arrondissement de Briey. Ces immigrés manifestent un vif attachement à leur langue, à leur culture, pour beaucoup à la foi catholique, et génèrent un riche tissu associatif. En vertu des accords franco-polonais de 1946, un rapatriement (ou une « réémigration » pour les autorités de Varsovie) est planifié sur la base du volontariat. Pour beaucoup, le choix entre la « mère patrie » et la " terre d'accueil » est délicat, voire douloureux ; il est fréquent qu'au sein d'une famille, parents et enfants se divisent. Les Polonais, placés au centre d'enjeux géopolitiques qui les dépassent, sont sensibles à la propagande émanant des associations favorables à la nouvelle Pologne (Parti ouvrier unifié polonais, communiste, « Aide à la patrie » ou « Grunwald »), ou sont, au contraire, à l'écoute des organisations catholiques ou fidèles à l'ancien gouvernement polonais de Londres. Les transferts les plus importants ont lieu de 1946 à 1948, et concernent trois à quatre mille personnes. Le rapatriement est pris en charge par la Croix-Rouge polonaise et par la SNCF qui organise des convois ferroviaires, de nature à en rappeler d'autres de sinistre mémoire. Les relations plus tendues entre les deux pays (démantèlement du réseau Robineau en Pologne en 1949) sont à l'origine de mesures répressives à l'égard des organisations favorables à la nouvelle démocratie populaire. Il est vrai que le traditionalisme religieux des Polonais ou leur exigence d'un enseignement particulier dans les écoles primaires communales contribuent à les rendre suspects tant aux yeux du clergé paroissial que des laïcs. Le départ des plus militants et la multiplication des naturalisations instaurent un climat plus serein à partir de 1950-51. 
Confrontés au rideau de fer, les catholiques ne restent pas indifférents. L’Église réitère sa condamnation du marxisme athée et dénonce de manière récurrente les "persécutions" exercées contre les croyants. L'évêque de Nancy, Mgr Fleury, fait lire en chaire une lettre de protestation contre les arrestations des primats de Hongrie et de Pologne (une des rares évocations de la Guerre froide constatée dans les bulletins paroissiaux). Les Lorrains sont attentifs aux déclarations du cardinal Tisserant, Nancéien d'origine et doyen du Sacré Collège, qui en tant que légat du pape, préside à Nancy en juillet 1949 le quatorzième Congrès eucharistique national ; à cette occasion, il est pris à partie par le PCF pour avoir affirmé que «la persécution est la conséquence de l’idéologie soviétique». Consciente que la déchristianisation du monde ouvrier facilite l'expansion du communisme, l’Église lance en 1947-48 des missions, et tente, sans grand enthousiasme, l'expérience des prêtres ouvriers. Ces religieux, au nombre de trois ou quatre seulement dans le département, analysent avec lucidité et perspicacité les mentalités ouvrières et les raisons de l'attrait exercé par les communistes. Ils soulignent l'importance de l'usine comme univers de vie quotidien, qui «nivelle, uniformise et endoctrine ${ }^{7}$, et qui est propice à faire naître les colères les plus explosives. L'usine est bien un véritable « laboratoire émotionnel ». Pour ce prolétariat, être communiste ne signifie pas adhérer à une pensée doctrinaire, mais « consiste à voir dans le parti la seule force capable d'améliorer la situation matérielle de la classe ouvrière ${ }^{8}$. Les préjugés règnent $:$ la papauté adhère au " parti américain »; donc le curé, qui vit bourgeoisement, est un "agent du capitalisme ». Il y a les bons et les méchants, et "le méchant est celui qui s'oppose à l'URSS »! L'Église doit donc chercher à reprendre pied dans l'usine ; pour cela, il convient de réactiver la doctrine sociale de l’Église, de faire appel aux associations catholiques laïques ("Action catholique ouvrière ", " Mouvement populaire des familles »). Pour certains militants catholiques, une collaboration avec la CGT est concevable, mais dans la seule optique de la lutte pour la dignité des travailleurs, comme cela s'est déjà produit lors des grèves de 1948. Sur le terrain politique, l'abbé Pierre, ancien résistant, élu député en 1945 sous l'étiquette MRP, affronte les communistes avec détermination, lors de réunions publiques et de cérémonies commémoratives, tout en partageant certains de leurs objectifs sociaux. Par ses propos chaleureux et son souci des difficultés matérielles, il force la sympathie de nombreux foyers ouvriers et brouille les clivages durcis par la Guerre froide.

7. ADMM, rapports des abbés Pfaff et Legendre, 51 J 1405/2 (Fonds Bonnet).

8. Ibid. 


\section{LA PRISE DE CONSCIENCE : \\ VERS UNE PERCEPTION PLUS AIGUË DES RÉALITÉS \\ DE LA GUERRE FROIDE}

La guerre et ses séquelles s'éloignant, l'opinion locale manifeste une écoute plus attentive aux problèmes du monde et se sent davantage concernée par les enjeux de la Guerre froide. L'expression apparaît dans l'été 1948, tant sous la plume des autorités que dans la presse : « c'est bien de la Guerre froide qu'il s'agit, et son champ de bataille est Berlin ${ }^{9}$. Les Lorrains n'observent que de loin la guerre d'Indochine, vue au travers du prisme colonial, mais dénoncée par le PCF comme la « sale guerre » et assimilée à la " guerre hitlérienne ». Plus tard, le désastre de Diên Biên Phu, à l'origine d'une vague de pessimisme, est l'occasion, le 8 mai 1954, d'associer, sur la base américaine de Toul, l'héroïsme des combattants français et l'implantation des forces de l'OTAN : le conflit indochinois est bel et bien devenu un conflit de la Guerre froide. La guerre en Corée, et l'établissement d'un autre rideau de fer le long du $38^{\mathrm{e}}$ parallèle conduisent la population à comprendre que l'affrontement ne se cantonne pas aux frontières européennes. L'opinion locale, selon les rapports préfectoraux, se « refuse à admettre que les Sud-Coréens soient les agresseurs, et se montre disposée à considérer comme légitime l'intervention des États-Unis ${ }^{10}$. Une sourde angoisse naît, d'une part, de la détermination américaine (menace de Mac Arthur d'utiliser l'arme atomique, rôle du général Ridgway, le «tueur microbien ») et, d'autre part, des réactions à attendre de Moscou et de Pékin. Plus consciente des dangers et du risque d'isolement de la France, l'opinion locale en vient à accepter le Pacte atlantique, présenté comme garant de la sécurité du pays, même si la CGT le considère comme « un plan de guerre, impérialiste, antisoviétique, anti-ouvrier et anti-démocratique ${ }^{11}$.

Mais comment se ranger aux vues américaines sans rien céder d'un anti-germanisme ambiant et traditionnel, allant de la plus vive hostilité, au sein de la droite nationale, à une méfiance prudente chez les modérés ? Déjà, l'épisode des péniches chargées de sucre à destination de la zone d'occupation française en Allemagne, et immobilisées par des manifestants à Verdun et à Frouard en septembre 1947, avait suscité une vive émotion, tout autant spontanée qu'orchestrée par les syndicalistes de la CGT et les communistes, et avivée des ressentiments antiallemands prompts à ressurgir. Alors que la construction d'une Europe intégrée n'intéresse guère que

9. La Croix de l'Est, $1^{\mathrm{er}}$ août 1948.

10. ADMM, Rapport du préfet, 18 août 1950, W 950/228.

11. ADMM, Tract, W 950/239. 
les démocrates-chrétiens, les socialistes ou quelques patrons d'industries, l'opinion locale est quasi unanime à se dresser contre l'éventualité d'un réarmement allemand et le projet de CED. Le PCF et ses alliés sont bien entendu les plus virulents à s'opposer au relèvement d'une Allemagne qu'ils qualifient de « réactionnaire, dirigée par les capitalistes et les anciens nazis ». La Voix de l'Est (journal du PC) n'affiche-t-elle pas une photo de la Wehrmacht accompagnée de la légende : "Qui veut revoir cela sur le cours Léopold $^{12}$ ? » Au début de 1954, se constitue un "Comité des Lorrains contre la CED », sorte d'alliance contre nature associant des gaullistes, des communistes et des nationalistes, et dont le président d'honneur est Louis Marin, président du Conseil général de 1946 à 1951 et de la Fédération républicaine, patriote intransigeant, que le préfet Samama qualifie «d'homme le plus représentatif des sentiments de la population » ${ }^{13}$. Le 27 décembre 1954, à l'exception du socialiste P.-O. Lapie, les cinq autres députés du département votent contre l'entrée de l'Allemagne dans l'OTAN, mais semblent désavoués par une partie de leurs électeurs. Comment assurer la sécurité du pays face au communisme sans l'aide américaine ? Comment accepter l'Alliance atlantique sans rien céder sur la question allemande ? Autant de contradictions que les citoyens locaux, qui mesurent mal le rôle désormais secondaire de la France, parviennent difficilement à surmonter.

L'éventualité d'un conflit majeur facilite l'éclosion du Mouvement de la paix, dont il convient de se demander s'il a été, et dans quelle mesure, un relais du parti communiste ? Les prémices en reviennent à la CGT et au PCF, qui lancent des appels en direction des autres syndicats et des associations patriotiques, en usant d'une argumentation portant sur la nécessité de défendre la paix face aux «horreurs» de la bombe A, quelles que soient les convictions politiques : « le problème actuel n'est pas d'être pour Moscou ou Washington, mais pour la guerre ou la paix ${ }^{14}$. La journée du « vote pour la paix » du 2 octobre 1949, sous forte surveillance policière, ne rencontre toutefois qu'un succès mitigé, mais la propagande s'amplifie en 1950 lorsque le PCF et la CGT incitent à signer l'Appel de Stockholm, et protestent contre la révocation de Joliot-Curie, le «savant de la paix », ou organisent le «relais de la jeunesse pour la paix » (en vue du festival de Berlin en 1951). Bien qu'interdits, deux Conseils communaux des combattants de la paix et de la liberté voient le jour ; à la fin de 1949, est constitué un Conseil départemental, présidé par le radical-socialiste Deville, mais au sein duquel se côtoient universitaires communistes, chrétiens progressistes et neutralistes. Auprès des associations patriotiques réceptives aux initiatives du Mouvement, le

12. La Voix de l'Est, 17 octobre 1953.

13. ADMM, Rapport du 8 septembre 1949, W 1131/62.

14. ADMM, RG Longwy 17 février 1949, W 950/95. 
combat pour la paix est assimilé au combat pour la Résistance, comme veulent le signifier les « Journées de la paix » organisées à Champigneulles, au cours desquelles les participants prêtent le "serment des partisans de la paix » devant la stèle rappelant le sacrifice de soixante-deux fusillés. Le meeting de Nancy du 3 février 1952 attire cinq mille personnes, en présence de prêtres (dont l'abbé Pierre), du grand rabbin et d'une délégation venue d'Oradour-sur-Glane. L'Appel de Stockholm embarrasse particulièrement les catholiques progressistes. Par la voix de l'évêque, Mgr Lallier, l’Église met en garde les fidèles et tente de réactiver le mouvement Pax Christi. Mais certains militants de l'Action catholique ouvrière, du Mouvement populaire des familles ou de la CFTC n'hésitent pas à donner leur signature, à l'instar de René Boudot, sidérurgiste qui représente le bassin de Longwy au Congrès des peuples pour la paix à Vienne en 1952. Nombre de curés des paroisses du Pays Haut sont directement sollicités par la CGT ; à celle-ci, qui fait valoir la présence d'hommes d'Église au sein du Mouvement, le curé de Trieux répond: "mais ce sont les communistes qui sont aux postes de direction ! ${ }^{15}$. Le préfet, de sensibilité socialiste, déclare, peut-être exagérément, que « la campagne pour la paix, et la signature de Stockholm n’ont eu aucun écho ${ }^{16}$. Sans aller jusque-là, on peut conclure à un large désintérêt des populations. Lors du procès des époux Rosenberg, les troupes américaines des bases et dépôts sont consignées par crainte de manifestations hostiles. Mais la population, du moins celle qui est informée, semble se rallier largement à la thèse de la culpabilité probable des « espions ». Un "Comité nancéien pour la défense des époux Rosenberg » sous l'impulsion d'israélites, associe à son action le Mouvement de la paix et Pax Christi, et lors du meeting du 15 juin 1953, le président du comité se contente de mettre l'accent « sur les conditions douteuses du procès », dans une salle décorée de drapeaux français et américains ${ }^{17}$.

Deux crises majeures vont plus particulièrement activer la prise de conscience de la Guerre froide, et renforcer une fois de plus le clivage idéologique. Lors des émeutes ouvrières de Berlin-Est, le syndicat FO s'empresse de rappeler que «lorsque les communistes sont au pouvoir, la classe ouvrière n'a plus aucun droit », lançant une polémique avec les militants communistes locaux. Avec la révolution de Hongrie, les Lorrains vivent véritablement au rythme de la Guerre froide, car, très rapidement, une large couverture médiatique sensibilise les populations locales. Les termes utilisés pour évoquer le peuple hongrois («rebelles », « insurgés ») et la répression («Saint Barthélémy rouge », «sanglante agonie de la liberté

15. ADMM, Police, W 950/95.

16. ADMM, Rapport de juillet 1950, W 950/228.

17. ADMM, RG, W 1131/29. 
hongroise ") ainsi que les images (chars soviétiques à Budapest, combats de rues) génèrent une forte charge émotionnelle, une vive sympathie pour les insurgés et une inquiétude certaine quant à la suite des événements. L'arrivée des réfugiés à l'automne 1956 transplante la tragédie hongroise au cœur des préoccupations quotidiennes ; de la fin de novembre 1956 à août 1957, le département accueille près de deux mille personnes, totalement démunies et logées provisoirement dans trois casernes. La confrontation subite avec ces fugitifs, jeunes pour la plupart, fatigués, inquiets, déclenche une réaction immédiate de compassion et d'empathie. Partout, des minutes de silence, des messes, des dépôts de gerbes aux monuments aux morts témoignent de la solidarité des populations locales à l'égard de la Hongrie martyrisée. Toutes les formes d'aide sont mises en œuvre, tant de la part des autorités que des entreprises ou des organisations politiques, syndicales et religieuses. L'indignation se retourne rapidement contre les communistes : comment croire, comme ceux-ci l'affirment, que ces réfugiés sont des fascistes antirévolutionnaires et des nostalgiques de Horthy ? Le 8 novembre à Nancy, un cortège conduit par un «Comité d'action universitaire», rassemble étudiants et représentants de la municipalité. Plusieurs milliers de personnes se recueillent devant le monument d'Alsace-Lorraine, puis la manifestation dégénère sous l'impulsion d'éléments radicaux (de tendance Algérie française) qui tentent de forcer les locaux du PCF et de la CGT. At-on cherché à instrumentaliser l'affaire hongroise en vue d'affaiblir un parti communiste, sorti renforcé des législatives de janvier ( $27 \%$ des voix) ? Toujours est-il que celui-ci est affecté par cette crise, de même que la CGT ; troublés, désorientés, certains militants essaient de susciter des débats internes, ou quittent leurs formations. Pourtant, un seul syndicat, la CGT des mines, prendra position publiquement contre l'intervention soviétique. La polémique perdure un moment, avec FO, avec la presse locale, notamment l'hebdomadaire Lorraine Magazine, qui prétend dévoiler « la vérité sur la Hongrie ${ }^{18}$, tandis que le PCF s'efforce de faire diversion en dénonçant la crise de Suez, la répression en Algérie et les questions sociales.

Les associations patriotiques de résistants et déportés n'échappent pas non plus au clivage Est/Ouest. Malgré des tensions croissantes, un relatif consensus se maintient toutefois entre ces associations, et nombre de cérémonies du souvenir les réunissent sous le signe de la fraternité : anniversaires de la Libération, transfert solennel des cendres de déportés au monument de la Résistance à Nancy (1950), hommage aux troupes américaines pour le vingtième anniversaire de la libération des camps. Au cours de l'année 1949, le consensus s'étiole et la polémique enfle ; face à la FNDIRP, largement menée par les communistes, la FNDIR soutient que

18. Lorraine Magazine, novembre 1956. 
« le titre de déporté ne peut figurer au crédit d'un seul parti politique » ${ }^{19}$. Aux propos du colonel Manhès, président national de la FNDIRP, qui affirme à Jarny-Homécourt que « ceux qui ont financé la guerre d'Hitler... préparent une nouvelle guerre ", répondent les gens de la FNDIR qui dénoncent la présence dans la haute administration de la RDA d'anciens déportés communistes qui " ont été les fidèles serviteurs des SS dans les camps $»{ }^{20}$. En 1950, la commission mise sur pied par David Rousset dans le but d'enquêter sur la persistance de camps de concentration, y compris en URSS, reçoit le soutien des seules FNDIR et associations " gaullistes ». Si les structures associatives sont saisies par la Guerre froide, le consensus mémoriel l'est aussi. Au-delà du souvenir, la mémoire devient un enjeu politique, avec ses rituels, ses lieux cérémoniels privilégiés : par exemple, le monument des fusillés de La Malpierre ou le camp de Thil pour la gauche, le monument de la Résistance à Nancy pour les gaullistes. Le PCF et ses satellites font référence aux dates mythiques $(1848,1934)$ et aux figures illustres de l'histoire... y compris Jeanne d'Arc et le général Giraud (mort en 1949) « qui n’a rien de commun avec tel général politicien qui n’a pas fait et n'a pas voulu faire la guerre... sauf au micro ${ }^{21}$. Au-delà de la mémoire, c'est l'histoire qui est révisée à la lumière de la Guerre froide, et sur ce point le cas de Victor Dojlida est emblématique. Ouvrier polonais, résistant $\mathrm{MOI} / \mathrm{FTP}$, rentré de déportation à l'âge de vingt ans, Dojlida est animé d'un désir de vengeance (de justice selon lui) à l'égard des gens de Vichy, patrons, policiers et juges, dont certains sont encore en place. Incapable de se réinsérer socialement, il multiplie les délits, malgré la sollicitude d'anciens compagnons de misère (dont le ministre Michelet). Condamné à plusieurs reprises, il est montré de moins en moins comme résistant, et déporté, mais comme un «bandit », voire "un étranger et un criminel ${ }^{22}$. Il ne sortira de prison qu'en 1989 ! Au début des années 1950, les Lorrains de Meurthe-et-Moselle sont conscients de l'existence de deux mondes différents et opposés, et l'opinion locale semble s'accommoder d'une certaine « normalité » de la Guerre froide.

19. Le Déporté résistant, février 1949, éditorial de Jean Bertin, président départemental.

20. Le Déporté résistant, février 1949.

21. La Voix de l'Est, 19 mars 1949.

22. L’Est Républicain, 29 avril 1948, plaidoirie de l'avocat général en Cour d'assises. 


\section{LA COHABITATION : \\ OU COMMENT S'ADAPTER À UNE CONFIGURATION MONDIALE JUGÉE DURABLE}

Du côté communiste, on cherche à établir une proximité plus étroite avec le monde soviétique et les démocraties populaires, tandis que les milieux modérés de droite et de gauche s'accommodent de l'appartenance au camp atlantique, et acceptent, voire souhaitent, la présence de forces militaires. Les communistes du département ont réussi à forger une véritable «mystique ouvrière », avec ses dogmes, ses valeurs, ses meetings, sa presse, ses slogans et ses héros, parmi lesquels Staline ${ }^{23}$ en personne. Son $70^{\mathrm{e}}$ anniversaire, puis sa mort seront l'occasion d'hommages grandioses, rendus avant tout au "vainqueur de Stalingrad ». Les gestes sincères et émouvants des militants (envoi de cadeaux, messages de reconnaissance) ne doivent pas occulter la mise en scène d'un culte de la personnalité orchestré par la Fédération. Au palais des fêtes de Nancy, le secrétaire Louis Dupont s'incline devant le portrait de Staline encadré d'une garde d'honneur : " nos yeux ne se détachent pas de ce visage exprimant la simplicité et la bonté » ${ }^{24}$. Le parti et ses partenaires, dont l'association France-URSS, propagent en Meurthe-et-Moselle comme dans toute la France, une vision héroïsée et idéalisée du monde communiste, grâce à la projection de films (Salut à Moscou, Oural, Cœur d'acier) ou par le biais de témoignages rapportés par des militants. Gilbert Schwartz, déporté politique, membre actif de la FNDIRP, dresse ainsi un tableau de la RDA, un pays « où avec quelle ardeur, avec quelle conscience chacun travaille, car chacun sait que l'usine est son usine, que c'est son avenir qu'il forge » ${ }^{25}$. Malgré les "dissensions internes » des années 1949-50, et l'émergence éphémère de tendances dissidentes, à l'encontre desquelles Laurent Casanova, membre du Comité central, de passage à Nancy, met en garde la Fédération, celle-ci reste fidèle à la ligne stalinienne. Selon le préfet Samama, les sanctions qui frappent Marty et Tillon «sont approuvées sans réserves par les sections et cellules » ${ }^{26}$. Dans son bastion du Pays Haut, les positions du PCF demeurent solides: $41 \%$ des voix dans le canton de Longwy en 1956, deux députés de 1956 à 1958. Le Parti s'est construit, parallèlement à son socle syndical, une assise municipale durable grâce à d'importantes victoires électorales, et la conquête de neuf municipalités dans le Pays Haut. Systématiquement, les élus

23. Montebello Fabrice, « Joseph Staline et Humphrey Bogart, l'hommage des ouvriers », Politix, n 24, 1993, p. 142.

24. La Voix de l'Est, 21 mars 1953.

25. La Voix de l'Est, 14-21 octobre 1950.

26. ADMM, Rapport du 12 janvier 1953, W 950/228. 
communistes introduisent des questions de politique générale, donc des enjeux de Guerre froide, dans les débats. Ces municipalités se bousculent pour tenter d'accueillir Khrouchtchev en 1960, et reçoivent avec fierté Alexis Leonov, le premier «piéton de l'espace », dont l'exploit spatial traduit « avant tout le succès du socialisme et du pouvoir des travailleurs ».

La pratique du jumelage vise, quant à elle, à «promouvoir l'amitié entre les peuples et la paix », mais aboutit souvent à pérenniser localement le climat de Guerre froide. Sur la période 1954-1967, on dénombre vingt-huit jumelages. Ceux qui sont conclus avec des communes de l'Est relèvent des années 1960 : huit communes (Auboué, Villerupt, Piennes...) signent neuf jumelages (huit avec la RDA, un avec la Tchécoslovaquie). L'initiative en revient souvent aux maires, et sept de ces jumelages sont placés sous le patronage de la Fédération mondiale des villes jumelées, qui se veut apolitique. Si les élus membres de la FNDIRP, du Mouvement de la paix et chrétiens progressistes approuvent l'initiative, les débats sont cependant houleux dans certains conseils : à Longlaville, les sept élus socialistes s’opposent au projet de jumelage. La pratique du jumelage facilitant « le déploiement des conflits idéologiques sur les scènes locales » 27 , les autorités font de l'obstruction systématique, refusent les visas d'entrée, en arguant du fait que la RDA n'est pas reconnue par la France, et que ces échanges n’ont aucune motivation culturelle, mais relèvent bien d'une « opération de caractère politique » ${ }^{28}$. Les jumelages fonctionnent donc «à sens unique »; en 1967, la délégation de Radebeul (RDA) n’est toujours pas autorisée à gagner Auboué, tandis que des délégations lorraines peuvent accéder à l'Europe de l'Est. Le préfet émet un rappel à l'ordre au maire de Longlaville, qui a fait arborer le drapeau de la RDA à l'occasion d'une exposition. Au-delà des aspects folkloriques, le jumelage implique clairement un engagement politique.

Dans les milieux modérés, majoritaires, la venue des Américains conforte-t-elle l'appartenance au camp occidental, voire une connivence sur des objectifs partagés ? Avec trois bases aériennes, dont deux opérationnelles (Toul-Rosières et Chambley), dotées d'armes nucléaires de 1956 à 1959, deux dépôts de l'US Army, et diverses autres installations, la Meurthe-et-Moselle est placée en première ligne du dispositif militaire de l'OTAN. Le retour des Américains (qui ne sont pas des inconnus depuis 1917 et 1944) ne suscite aucune hostilité déclarée, ni à l'égard de l'Alliance atlantique, ni à l'encontre des soldats américains. Seules les expropriations et la lenteur des indemnisations provoquent un certain mécontentement ; mais, rapidement, les retombées économiques et financières

27. Vion Antoine, «L'invention de la tradition des jumelages », Revue française de science politique, vol. 53, $\mathrm{n}^{\circ} 4$, 2003, p. 570.

28. AN, Fontainebleau, versement 19780663. 
deviennent intéressantes pour les entreprises locales, les commerçants et les propriétaires de logements. La croissance des effectifs et l'arrivée des familles (plus de onze cents pour la base de Rosières) nécessitent la construction de cités pavillonnaires à l’urbanisme américain : Toulaire, Regina Village... En termes d'emplois, le seul secteur de Toul ira jusqu'à compter 3400 salariés civils. La présence américaine est également à l'origine d'un véritable choc culturel. Durant les premières années, les contacts entre Américains et Français sont réduits et sans chaleur particulière, d'autant que les bases vivent en totale autarcie. Les Lorrains sont d'ailleurs mal informés : " pourquoi sont-ils là, que viennent-ils faire ?, c'est parce que nous les avons appelés à nous aider ${ }^{29}$. Chez les Américains, il convient de surmonter les préjugés à l'égard des Français, et de répondre à leurs récriminations à propos du bruit des avions, des trop nombreux accidents de la route, des rixes sur la voie publique ou dans les débits de boissons. Mais, très vite, s'affirme une volonté réciproque de compréhension et de coopération, soutenue par l'attrait qu'exerce le mode de vie américain et le désir d'ouverture que manifestent certains officiers américains francophiles, surtout chez les réservistes de la National Air Guard arrivés en 1961. Fêtes nationales et cérémonies militaires sont prétextes à vanter la fraternité d'armes et l'amitié franco-américaine ; lors des défilés, la foule applaudit ceux qui sont présentés comme «nos alliés ». Le point d'orgue du travail de séduction est atteint avec les « journées portes ouvertes » sur les bases; le 18 juin 1961, entre 50000 et 75000 spectateurs se pressent au meeting aérien de Rosières. La présence d'un Civiliant Consultant français sur les bases, chargé des contacts extérieurs contribue à « dépouiller les relations franco-américaines de leur raideur officielle ${ }^{30}$. Mieux encore, les nombreux mariages mixtes ne sont-ils pas la meilleure expression de la proximité relationnelle entre Français et Américains ?

La population locale considère, à l'instar des gouvernements, que l'appartenance à l'Alliance atlantique ne doit en aucun cas limiter l'indépendance et la souveraineté nationales. Le rôle de l'officier de liaison, à la tête d'un détachement restreint, est justement d'être le gardien vigilant de la souveraineté territoriale sur des bases qui ne peuvent être perçues comme des enclaves étrangères. De ce fait, des litiges éclatent à propos des contrôles douaniers, de la circulation des étrangers et des compétences juridictionnelles respectives concernant les crimes et délits. D'ailleurs, les multiples manifestations d'entente et de sympathie ne sont pas appréciées de tous. Catholiques et communistes se rejoignent parfois dans le même rejet du cinéma américain, jugé immoral et violent. Le curé

29. L'Est Républicain, 14 décembre 1951.

30. ADMM, rapport de Chambley, décembre 1955, W 950/41. 
de Rosières fait savoir son indignation à ses paroissiens, quand des jeunes filles de la localité participent à une fête à la base, une semaine après Diên Biên Phu, "alors que des Français sont tués, blessés, prisonniers... hommes de Rosières, on vous a pris vos champs, ne permettez pas que l'on s'amuse avec vos femmes et vos jeunes filles " ${ }^{31}$. La méconnaissance des objectifs et des dispositifs de l'OTAN laisse le champ libre à la propagande hostile du PCF. Selon le préfet, seuls les communistes se signalent : tracts, affiches, graffitis US Go Home fleurissent dans les années 1950-52, avant de réapparaître vers 1965-66. Ceux-ci ne cessent de dénoncer « une armée d'occupation », vouée à faire du département une "terre brûlée», une " poudrière » et une plate-forme d'attaque contre l'URSS. Rien cependant ne permet de conclure à un impact mesurable de cette propagande dans la progression (relative) des communistes au cours des élections de 1953 et 1956. Après quelques années d'assoupissement qui succèdent à l'alerte maximale de 1956, les bases se réveillent au moment des crises de Berlin et de Cuba ; mais les missions nucléaires sont abandonnées au profit de la reconnaissance aérienne et du soutien aux forces stationnées en Allemagne. Peu diserts sur les questions politiques, les Américains se cantonnent dans une stricte neutralité. Anticolonialistes affichés, ils redoutent néanmoins la victoire d'un FLN « acquis au communisme ». Ils sont surpris, voire dépités devant le succès populaire rencontré par Khrouchtchev lors de sa venue dans la région. À l'égard de De Gaulle, leurs sentiments sont partagés : réserve, incompréhension, mais aussi respect et considération. Lorsque les Américains quittent la Lorraine, laissant un marché de l'emploi gravement déprimé, c'est avec un regret partagé, et « on a l’impression que leur départ entraîne un resserrement des liens franco-américains ${ }^{32}$. C'est le moment choisi pour la visite du général Lemnitzer, commandant du SHAPE, venu à Nancy inaugurer un viaduc auquel est donné le nom de Kennedy ; le maire Weber remercie les Américains en termes chaleureux. Leur souvenir restera associé à l’image de prospérité laissée par les « Trente Glorieuses »

L'adaptation à l'état de Guerre froide semble se traduire par une modification des appréhensions suscitées par les événements. L’opinion locale paraît passer d'un réflexe de peur irrationnelle à une appréhension plus raisonnée et mieux maîtrisée. Durant ces vingt années, ce sont plutôt des émotions successives, des angoisses ponctuelles, qui surgissent sur un fonds d'indifférence assez large et d'inquiétude latente. La guerre de Corée, les crises de Hongrie et de Cuba sont propices à des dynamiques émotionnelles qui ne conduisent qu'exceptionnellement à des phénomènes de panique. En 1950, La Voix de l'Est publie une photo montage de la place

31. ADMM, RG 11 mai 1954, W 950/276.

32. ADMM, rapport de Chambley, juin 1966, W 950/41. 
Stanislas sous un champignon atomique, évoquant Hiroshima. En 1961, Le Sous-sol lorrain, organe de la CGT, titre « La guerre atomique pour Berlin ? » En réalité, la population redoute plus « une guerre » qu'une conflagration atomique. Si le PCF joue de la peur atomique, le Mouvement de la paix ou l'Église montrent un souci réel d'information : des conférences abordent les implications scientifiques et morales de l'arme atomique. Il semble qu'avec la présence des forces de l'OTAN, puis la politique gaullienne d'indépendance nationale (appuyée sur une force nucléaire), la peur du danger atomique s'atténue et fasse place à un sang-froid lucide, dans un contexte moins tendu, celui de la coexistence pacifique. « La survie pour 7000 francs par personne » annonce une publicité vantant les qualités d'un abri antiatomique à la foire de Nancy en 1964. La Guerre froide ne seraitelle devenue qu'un banal objet de consommation?

La Guerre froide n'est donc pas passée inaperçue des Lorrains, mais la nature exacte et l'ampleur de son ressenti sont difficilement mesurables. Les rapports des autorités officielles n'accordent qu'une place secondaire aux réactions de la population locale face aux événements internationaux. Celle-ci prend conscience rapidement de la nouvelle conjoncture mondiale, sans percevoir pleinement la complexité des enjeux, et tout en s'illusionnant sur l'influence réelle de la France. Les Lorrains, en priorité concernés par les séquelles de la guerre et attentifs aux questions coloniales, ne sont pas indifférents à la confrontation entre les deux blocs, de plus en plus fréquemment à « la Une » des quotidiens. Parallèlement à un vif ressenti émotionnel au moment des crises les plus aiguës, mais qui ne va pas jusqu'à la peur physique, existe une appropriation plus analytique, plus « intellectuelle », perceptible au travers des éditoriaux ou des discours des acteurs locaux. Les comportements individuels vont de l'indifférence à la curiosité, de l'inquiétude à l'angoisse, du recueillement à la violence, au gré de la gravité des événements, ainsi que de l'exploitation médiatique et politique qui en est faite. La désinformation, la propagande, la manipulation, l'utilisation de l'image participent à la construction de ce ressenti, en proposant alors une interprétation faussée, partiale, sectaire des événements. Il est évident que le clivage entre modérés et communistes, tout à la fois cause et conséquence de ce ressenti, ainsi que la présence américaine ou l'arrivée des réfugiés hongrois proposent des visions divergentes des deux blocs et incitent les Lorrains (Français et étrangers) à s'interroger sur les choix à opérer. Pour autant, la Guerre froide ne semble pas avoir influencé au point de la modifier une configuration politique largement déterminée par les questions nationales et locales. 